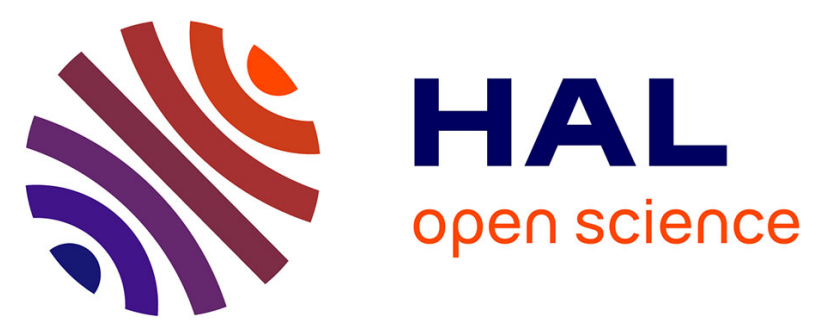

\title{
Incidence and Outcome of Sub-clinical Acute Kidney Injury Using penKid in Critically Ill Patients
}

François Dépret, Alexa Hollinger, Alain Cariou, Nicolas Deye, Antoine Vieillard-Baron, Marie-Céline Fournier, Samir Jaber, Charles Damoisel, Qin $\mathrm{Lu}$, Xavier Monnet, et al.

\section{To cite this version:}

François Dépret, Alexa Hollinger, Alain Cariou, Nicolas Deye, Antoine Vieillard-Baron, et al.. Incidence and Outcome of Sub-clinical Acute Kidney Injury Using penKid in Critically Ill Patients. American Journal of Respiratory and Critical Care Medicine, 2020, 202 (6), pp.822-829. 10.1164/rccm.201910-1950OC . hal-02864580

\section{HAL Id: hal-02864580 \\ https://hal.science/hal-02864580}

Submitted on 28 Oct 2020

HAL is a multi-disciplinary open access archive for the deposit and dissemination of scientific research documents, whether they are published or not. The documents may come from teaching and research institutions in France or abroad, or from public or private research centers.
L'archive ouverte pluridisciplinaire HAL, est destinée au dépôt et à la diffusion de documents scientifiques de niveau recherche, publiés ou non, émanant des établissements d'enseignement et de recherche français ou étrangers, des laboratoires publics ou privés. 


\section{Incidence and Outcome of Subclinical Acute Kidney Injury Using penKid in Critically III Patients}

François Dépret ${ }^{1,2,3,4,5}$, Alexa Hollinger ${ }^{1,2,6}$, Alain Cariou ${ }^{7}$, Nicolas Deye ${ }^{2,8}$, Antoine Vieillard-Baron ${ }^{9,10,11}$, Marie-Céline Fournier ${ }^{1,2,5}$, Samir Jaber ${ }^{12}$, Charles Damoisel ${ }^{1}$, Qin Lu ${ }^{13,14}$, Xavier Monnet ${ }^{15,16,17}$, Isabelle Rennuit ${ }^{18}$, Michael Darmon ${ }^{4,19,20}$, Marc Leone ${ }^{21,22}$, Bertrand Guidet ${ }^{14,23}$, Romain Sonneville ${ }^{4,24}$, Philippe Montravers ${ }^{4,25}$, Sébastien Pili-Floury ${ }^{26,27}$, Jean-Yves Lefrant ${ }^{28,29,30}$, Jacques Duranteau ${ }^{31}$, Pierre-François Laterre ${ }^{32}$, Nicolas Brechot ${ }^{33}$, Haikel Oueslati ${ }^{1}$, Bernard Cholley ${ }^{34,35}$, Joachim Struck ${ }^{36}$, Oliver Hartmann ${ }^{36}$, Alexandre Mebazaa ${ }^{1,2,3,4,5}$, Etienne Gayat ${ }^{1,2,3,4,5}$, and Matthieu Legrand ${ }^{4,37}$

${ }^{1}$ Department of Anaesthesiology, Critical Care Medicine and Burn Unit, Saint Louis-Lariboisière University Hospitals, Assistance Publique-Hôpitaux de Paris (AP-HP), Paris, France; ' ${ }^{2}$ MMR-S942, Institut National de la Santé et de la Recherche Médicale (INSERM), Lariboisière Hospital, Paris, France; ${ }^{3}$ INI-CRCT Network, Paris, France; ${ }^{4}$ Paris University, Paris, France; ${ }^{5}$ INSERM 942, FHU Promice, Paris, France; ${ }^{6}$ Department of Anesthesia, Surgical Intensive Care, Prehospital Emergency Medicine and Pain Therapy, University Hospital Basel, Basel, Switzerland; ${ }^{7}$ Medical Intensive Care Unit, Cochin University Hospital, AP-HP, Paris, France; ${ }^{8}$ Medical Intensive Care Unit, Saint Louis-Lariboisière University Hospitals, AP-HP, Paris, France; 9 Intensive Care Unit, University Hospital Ambroise Paré, AP-HP, Boulogne-Billancourt, France; ${ }^{10}$ INSERM U-1018, CESP, Team 5, University of Versailles, Saint-Quentin en Yvelines, France; ${ }^{11}$ Faculty of Medicine Paris lle-de-France Ouest, University of Versailles, Saint-Quentin en Yvelines, France; ${ }^{12}$ Intensive Care Unit, Anesthesia and Critical Care Department, Saint Eloi Teaching Hospital, Montpellier, France; ${ }^{13}$ Multidisciplinary Intensive Care Unit, Department of Anesthesiology and Critical Care Medicine, La Pitié-Salpétrière Hospital, AP-HP, Paris, France; ${ }^{14}$ Pierre and Marie Curie University, Paris 6, Paris, France; ${ }^{15}$ Medical Intensive Care Unit, Bicêtre Hospital, Paris-Sud University Hospitals, Le Kremlin-Bicêtre, France; ${ }^{6}{ }_{1}$ INSERM UMR S999, Paris, France; ${ }^{17}$ Paris-Sud 5 University, Orsay, France; ${ }^{18}$ Department of Anesthesiology and Critical Care, Beaujon Hospital, AP-HP, Clichy, France; ${ }^{19}$ Medical Intensive Care Unit, Saint-Louis Hospital, AP-HP, Paris, France; ${ }^{20}$ ECSTRA Team, Biostatistics and Clinical Epidemiology, UMR 1153 (Center of Epidemiology and Biostatistics Sorbonne Paris Cité, CRESS), INSERM, Paris, France; ${ }^{21} \mathrm{APHM}$, Department of Anesthesiology and Critical Care Medicine, Marseille, France; ${ }^{22}$ Aix Marseille University,

Marseille, France; ${ }^{23}$ Medical Intensive Care, Saint-Antoine Hospital, AP-HP, Paris, France; ${ }^{24}$ Department of Intensive Care Medicine and Infectious Diseases, Bichat-Claude Bernard Hospital, AP-HP, Paris, France; ${ }^{25}$ Department of Anesthesiology and Intensive Care, Bichat University Hospital, AP-HP,

Paris, France; ${ }^{26}$ Department of Anesthesiology and Intensive Care Medicine, Besançon University Hospital, EA 3920, Besançon, France; ${ }^{27}$ University of Bourgogne Franche-Comté, Besançon, France; ${ }^{28}$ Department of Anesthesiology, Critical Care, Emergency and Pain Medicine, Nimes University Hospital, Nimes, France; ${ }^{29}$ Research Unit EA 2992, Nimes, France; ${ }^{30}$ Montpellier 1 University, Nîmes, France; ${ }^{31}$ Department of Anesthesiology and Critical Care, ParisSud, AP-HP, Le Kremlin-Bicêtre, France; ${ }^{32}$ Department of Critical Care Medicine, Saint Luc University Hospital, Catholic University of Louvain, Brussels, Belgium; ${ }^{33}$ INSERM, U833, Angiogenesis, Embryogenesis and Pathology Laboratory, Collège de France, Paris, France; ${ }^{34}$ Department of Anesthesiology and Intensive Care, European Hospital Georges Pompidou University Hospital, AP-HP, Paris, France; ${ }^{35}$ Paris Descartes University-Paris 5, Paris, France; ${ }_{36}$ sphingotec $\mathrm{GmbH}$, Hennigsdorf, Germany; and ${ }^{37}$ Department of Anesthesia and Perioperative Care, University of California San Francisco, San

Francisco, California

\section{Abstract}

Rationale: Subclinical acute kidney injury (sub-AKI) refers to patients with low serum creatinine but elevated alternative biomarkers of AKI. Its incidence and outcome in critically ill patients remain, however, largely unknown. Plasma proenkephalin A 119-159 (penKid) has been proposed as a sensitive biomarker of glomerular function.

Objectives: In this ancillary study of two cohorts, we explored the incidence and outcome of sub-AKI based on penKid.

Methods: A prospective observational study in ICUs was conducted. FROG-ICU (French and European Outcome Registry in ICUs) enrolled 2,087 critically ill patients, and AdrenOSS-1 (Adrenomedullin and Outcome in Severe Sepsis and Septic Shock-1) enrolled 583 septic patients. The primary endpoint was 28 -day mortality after ICU admission. Sub-AKI was defined by an admission penKid concentration above the normal range (i.e., $>80 \mathrm{pmol} / \mathrm{L}$ ) in patients not meeting the definition of AKI. A sensitivity analysis was performed among patients with estimated glomerular filtration rate above $60 \mathrm{ml} / \mathrm{min} / 1.73 \mathrm{~m}^{2}$ at ICU admission.
Measurements and Main Results: In total, 6.1\% (122/2,004) and $6.7 \%(39 / 583)$ of patients from the FROG-ICU and AdrenOSS-1 cohorts met the definition of sub-AKI (11.6\% and $17.5 \%$ of patients without $\mathrm{AKI}$ ). In patients without AKI or with high estimated glomerular filtration rate, penKid was associated with higher mortality (adjusted standardized hazard ratio [HR], 1.4 [95\% confidence interval, 1.1-1.8]; $P=0.010$; and HR, 1.6 [95\% confidence interval, 1.3-1.8]; $P<0.0001$, respectively) after adjustment for age, sex, comorbidities, diagnosis, creatinine, diuresis, and study. Patients with sub-AKI had higher mortality compared with no AKI (HR, 2.4 [95\% confidence interval, 1.5-3.7] in FROG-ICU and 2.5 [95\% confidence interval, 1.1-5.9] in AdrenOSS-1).

Conclusions: Sub-AKI defined using penKid occurred in $11.6-17.5 \%$ of patients without AKI and was associated with a risk of death close to patients with AKI.

Keywords: acute kidney injury; renal recovery; biomarker; proenkephalin; penKid 


\section{Scientific Knowledge on the}

Subject: Subclinical acute kidney injury (sub-AKI) refers to patients with low serum creatinine but elevated alternative biomarkers of AKI. Its incidence and outcome in critically ill patients remain, however, largely unknown. Plasma proenkephalin A 119-159 (penKid) has been proposed as a sensitive biomarker of glomerular function.

\section{What This Study Adds to the Field:}

In two cohorts of 2,087 and 583 critically ill patients, respectively, subAKI defined using penKid occurred in $11.6 \%$ to $17.5 \%$ of patients without AKI. In patients without AKI or with high estimated glomerular filtration rate at admission, penKid was associated with higher mortality. SubAKI was associated with a risk of death close to patients with AKI.

The current definition of acute kidney injury (AKI) in the medical literature is issued by the Kidney Disease: Improving Global Outcomes (KDIGO) group. This definition is based on acute variations of serum creatinine (Screat) and/or low urine output (1). There is, however, a consensus on the poor sensitivity of these biomarkers in acute settings (2). Screat may lack sensitivity to detect changes in glomerular filtration rate in severely ill patients. The lack of sensitivity of Screat is mostly due to its large variability and the decreased production of creatinine in patients with sepsis or low muscular mass (3). In addition, the rise in Screat is delayed and occurs only when a substantial part $(\sim 50 \%)$ of the renal functional reserve has been affected (4). The term "subclinical AKI" (sub-AKI) has been proposed to describe the occurrence of kidney damage or dysfunction without meeting standard definition of AKI and based on detection of alternative biomarkers of AKI (5). The incidence and outcome of sub-AKI in critically ill patients remain, however, largely unknown. Furthermore, whether detecting sub-AKI would be relevant with respect to outcome remains elusive.

In this study, we explored the incidence and outcome of sub-AKI in critically ill patients using plasma proenkephalin A 119-159 (penKid). penKid has been proposed as a sensitive biomarker of glomerular function. As such it was found to be predictive for septic kidney events, including AKI, in different settings (6-8). Our primary aim was to investigate the potential association between sub-AKI and short-term outcome in two large cohorts of ICU patients.

\section{Methods}

\section{Study Design}

The FROG-ICU (French and European Outcome Registry in ICUs) cohort served as an exploratory cohort (9). The AdrenOSS-1 (Adrenomedullin and Outcome in Severe Sepsis and Septic Shock-1) cohort enrolled 583 patients with severe sepsis or septic shock (10). The FROG-ICU study (clinicaltrials.gov identifier: NCT01367093) was a European prospective, observational, and multinational study in 24 centers from five countries (France, Belgium, the Netherlands, Italy, and Germany), including 2,087 ICU consecutive patients. Patients were recruited from June 2015 to May $2016(9,10)$. Results for the association of penKid with AKI, major adverse kidney events, worsening renal function, and 28-day mortality have already been published elsewhere (7).

\section{Endpoint Definition}

AKI definition was based on the KDIGO criteria that use Screat as previously described
$(7,11,12)$. Baseline Screat was assessed using the following method. If estimated glomerular filtration rate (eGFR) on admission was $\geqslant 75 \mathrm{ml} / \mathrm{min}$ per $1.73 \mathrm{~m}^{2}$, we used the actual Screat on admission (admission Screat). If eGFR was $<75 \mathrm{ml} / \mathrm{min}$ per $1.73 \mathrm{~m}^{2}$ on admission, we used the Screat calculated back from the MDRD (Modification of Diet in Renal Disease Study) equation set to $75 \mathrm{ml} / \mathrm{min}$ per $1.73 \mathrm{~m}^{2}$.

Sub-AKI was defined by plasma penKid $>80 \mathrm{pmol} / \mathrm{L}$ in patients without AKI (defined with the KDIGO criteria, i.e., non$\mathrm{AKI}_{\mathrm{KDIGO}}$ but with an abnormal penKid level) (7). Threshold was chosen as the 99th percentile of penKid plasma value in healthy volunteers (8). As a sensitivity analysis, we explored the association between penKid at admission and mortality in patients with eGFR above 60 $\mathrm{ml} / \mathrm{min} / 1.73 \mathrm{~m}^{2}$ at ICU admission.

\section{Measurement of penKid}

Blood for the central laboratory was sampled within 24 hours after admission. Samples were subsequently processed and stored at $-80^{\circ} \mathrm{C}$ before transfer to the central laboratory for the blinded penKid analysis. The method of measurement, lower detection limit, and intraassay and interassay coefficients of variation have been published elsewhere (7). penKid has been measured with a commercially available chemiluminescence immunoassay (SphingoTec GmbH). Both intraassay and interassay coefficients of variation as well as interindividual variability (biological variation) for penKid with a commercially available chemiluminescence immunoassay (SphingoTec $\mathrm{GmbH}$ ) are reported to be low. Intraassay and interassay coefficients of variation were $6.4 \%$ and $9.5 \%$ at $50 \mathrm{pmol} / \mathrm{L}$ and $4.0 \%$ and $6.5 \%$ at 150 $\mathrm{pmol} / \mathrm{L}$, respectively.

\section{Endpoints}

The primary endpoint was 28-day allcause mortality. Secondary endpoint was

AdrenOSS-1 (ClinicalTrials.gov identifier NCT02393781) was sponsored by Sphingotec GmbH, Neuendorfstraße. This project was partially funded by the European Union's Horizon 2020 research and innovation program (grant 666328). FROG-ICU (French and European Outcome Registry in ICUs) was funded by the Program Hospitalier de la Recherche Clinique (AON 10-216) and by a research grant from the Société Française d'Anesthésie-Réanimation.

Author Contributions: O.H. and E.G. performed statistical analysis. F.D., A.H., A.C., N.D., A.V.-B., M.-C.F., S.J., C.D., Q.L., X.M., I.R., M.D., M. Leone, B.G., R.S., P.M., S.P.-F., J.-Y.L., J.D., P.-F.L., N.B., H.O., B.C., J.S., O.H., A.M., and E.G. collected data, corrected the first version of the manuscript, and approved the final version. M. Legrand conceived the study, drafted the manuscript, and approved the final version.

Correspondence and requests for reprints should be addressed to Matthieu Legrand, M.D., Department of Anesthesia and Perioperative Care, 500 Parnassus Avenue MUE416, Box 0648, University of California San Francisco, San Francisco, CA 94143. E-mail: matthieu.legrand@ucsf.edu. 
hemodynamic organ support within the first week (defined as the need for intravenous norepinephrine, epinephrine, dobutamine, or dopamine).

\section{Collection of Patient Data}

Upon admission, demographics (age and sex), body mass index, presence of sepsis or septic shock, type of ICU admission, organ dysfunction and severity scores (Sequential Organ Failure Assessment [SOFA] and Acute Physiology and Chronic Health Evaluation II score), the source of sepsis, preexisting comorbidities treated within the past year, past medical history, laboratory values, urine output, and organ support were recorded. During the first week after patient enrollment, the following data were assessed and/or collected on a daily basis: SOFA score, antimicrobial therapies, fluid balance, ventilation status, Glasgow Coma Scale score, need for renal replacement therapy (RRT), invasive procedures for sepsis control, and vasopressor treatment. On Day 28, discharge status or mortality was recorded in all study participants. Baseline kidney function was not part of inclusion or exclusion criteria in both studies.

\section{Statistical Analysis}

Values are expressed as medians (interquartile ranges $[\mathrm{IQR}]$ ) or counts (percentages), as appropriate. Group comparisons of continuous variables were performed using the Kruskal-Wallis test. Categorical data were compared using Pearson's chi-squared test for count data. penKid data were log-transformed when analyzed as a continuous variable. Cox proportional hazards regression was used to analyze the effect of penKid on survival. For multivariate analyses, data from both studies were combined. penKid association with primary endpoint was adjusted for age, sex, comorbidities (cardiac and noncardiac), diagnosis (septic shock vs. other), creatinine and diuresis in the past 24 hours (variables significant for mortality in univariate analysis in patients without AKI), and study. When analyzed as a continuous variable, hazard ratios (HRs) (95\% confidence intervals) were standardized to describe the HR for a biomarker change of one IQR. Survival curves plotted by the Kaplan-Meier method were used for illustrative purposes. As a sensitivity analysis, we applied receiver operating characteristic (ROC) analysis to determine the optimal cut point based on the Youden criteria for sub-AKI definition to compare these to our prespecified cut point of $80 \mathrm{pmol} / \mathrm{L}$.

All statistical tests were two-tailed, and a two-sided $P$ value of 0.05 was considered for significance. The statistical analyses were performed using $\mathrm{R}$ version 3.4.3 (http://www.r-project.org, library Design, Hmisc, ROCR) and Statistical Package for the Social Sciences version 22.0 (SPSS Inc.).

\section{Results}

Two thousand four patients from the FROG-ICU cohort and all 583 patients from the AdrenOSS- 1 cohort were eligible for data analysis. The 28 -day mortality was $22 \%$ in both cohorts. In total, 951 (47.2\%) and $360(61.7 \%)$ patients from FROG-ICU and AdrenOSS-1 had AKI, including 587 (29.1\%) in the FROG-ICU and 101 (17.3\%) in AdrenOSS-1 with severe AKI; $23.3 \%$ and $15.4 \%$ received RRT, respectively.

\section{Clinical Characteristics of Sub-AKI}

Among the patients who did not develop AKI based on the KDIGO definition, $11.6 \%$ $(122 / 1,056)$ in the FROG-ICU cohort and $17.5 \%(39 / 223)$ in the AdrenOSS-1 cohort were classified as having sub-AKI based on penKid $>80 \mathrm{pmol} / \mathrm{L}$. This represented $6.1 \%$ $(122 / 2,004)$ and $6.7 \%(39 / 583)$ of all patients enrolled in FROG-ICU and AdrenOSS-1, respectively. Median SOFA score was similar in patients without AKI and those with subAKI in the FROG-ICU cohort and slightly higher in patients with sub-AKI in the AdrenOSS-1 cohort (median, 6 [IQR, 6-9] vs. 5 [IQR, 3-7]; $P=0.011$ ). Characteristics of all patients and by subgroups, non-AKI, sub-AKI, and AKI, are summarized in Tables 1 and 2. Patients classified as having sub-AKI, compared with patients without AKI, were more likely to have septic shock and more comorbidities (cardiac and noncardiac) (Tables 1 and 2).

\section{Sub-AKI and Mortality}

Over a 28-day follow-up period, 441 patients $(22.0 \%)$ died in the FROG-ICU cohort and 127 patients $(21.8 \%)$ died in the AdrenOSS-1 cohort. There was a significant stepwise decrease of 28-day survival from patients without AKI $(90.8 \%$ [ $95 \%$ confidence interval, 89.0-92.7]) to those with sub-AKI (79.5\% [72.7-87.0]) and AKI (65.2\% [62.2-68.3]) in the FROG-ICU cohort as well as in the AdrenOSS-1 cohort (91.3\%
[86.2-94.6], 78.9\% [62.3-88.9], and 71.3\% [66.3-75.7], respectively) (Figure 1). The HR for patients with sub-AKI versus those without sub-AKI was 2.4 (1.5-3.7) in FROG-ICU and 2.5 (1.1-5.9) in AdrenOSS-1 (Figure 2).

In patients not meeting the KDIGO definition for AKI, 28-day survival was 89.5\% ( $n=111$ events) and 89.2\% $(n=24$ events) in FROG-ICU and AdrenOSS-1, respectively. In these patients, penKid (as continuous variable) was associated with 28-day mortality in both cohorts (standardized HR, 1.6 [1.3-2.0]; $P<0.0001$; c index, 0.604 [0.549-0.660]; $P<0.0001$; and HR, 1.7 [1.0-2.7]; $P=0.04 ; c$ index, 0.606 [0.486-0.727]; $P=0.0375$,

respectively) (Figure 2), whereas creatinine was only significant in the FROG-ICU cohort (HR, 1.3 [1.0-1.7]; $P=0.030 ; c$ index, 0.569 [0.514-0.625]; $P=0.0300$; and HR, 1.4 [0.7-2.6]; $P=0.337$; $c$ index, 0.587 [0.462-0.713]; $P=0.3374$, respectively). C index for urine output to predict mortality was $0.488(0.369-0.608 ; P=0.710)$ in the AdrenOSS-1 cohort and 0.561 (0.506-0.617; $P=0.019)$ in the FROG-ICU cohort, respectively.

In the combined population without $\mathrm{AKI}$, in a Cox proportional hazard model adjusted for age, sex, cardiac comorbidities, noncardiac comorbidities, diagnosis, creatinine, diuresis, and study, penKid concentration on admission was independently associated with 28-day mortality (adjusted standardized HR, 1.4 [1.1-1.8]; $P=0.010)$. C index increased from 0.673 to $0.682(P=0.012)$. In patients with eGFR above $60 \mathrm{ml} / \mathrm{min} / 1.73 \mathrm{~m}^{2}$, penKid concentration on admission was also independently associated with 28-day mortality (adjusted standardized HR, 1.6 [1.3-1.8]; $P<0.0001 ; c$ index increased from 0.677 to $0.702 ; P<0.0001$ ). In both patients without AKI or eGFR $>60$ $\mathrm{ml} / \mathrm{min} / 1.73 \mathrm{~m}^{2}$, creatinine was not significant in the multivariable model ( $P=0.888$ and $P=0.939$, respectively).

ROC analysis for 28-day mortality in patients without AKI resulted in an area under the curve of $0.61(0.55-0.67)$, $0.58(0.52-0.63)$, and $0.55(0.49-0.60)$ for penKid, creatinine, and diuresis, respectively $(P<0.0001,0.0228$, and 0.0250 , respectively). In patients with eGFR $>60 \mathrm{ml} / \mathrm{min} / 1.73 \mathrm{~m}^{2}$, areas under the curve were $0.66(0.61-0.70), 0.57(0.52-0.61)$, and $0.61(0.57-0.66)$ for penKid, creatinine, and diuresis, respectively $(P<0.0001,0.0575$, and 0.0001 , respectively). In both 


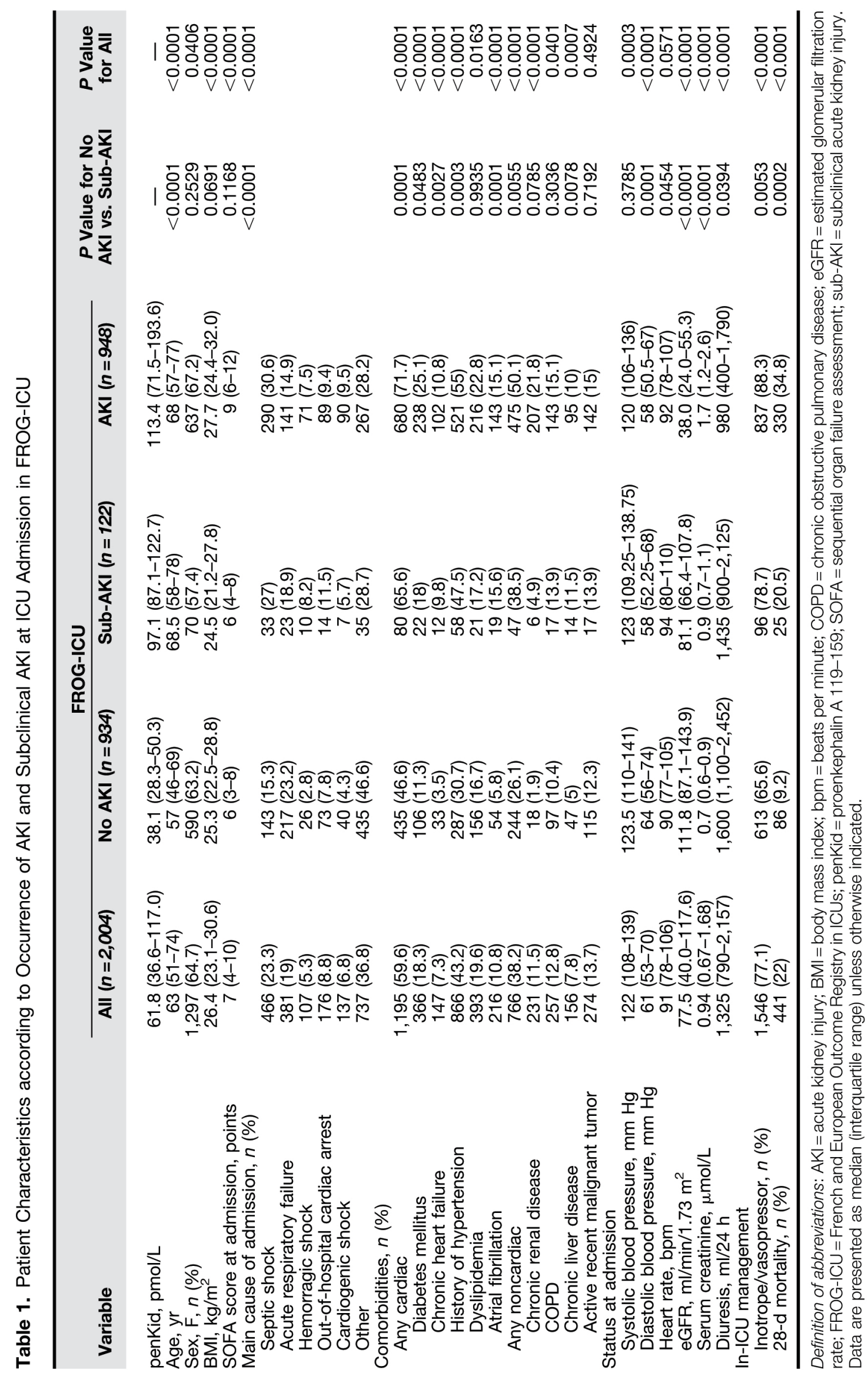




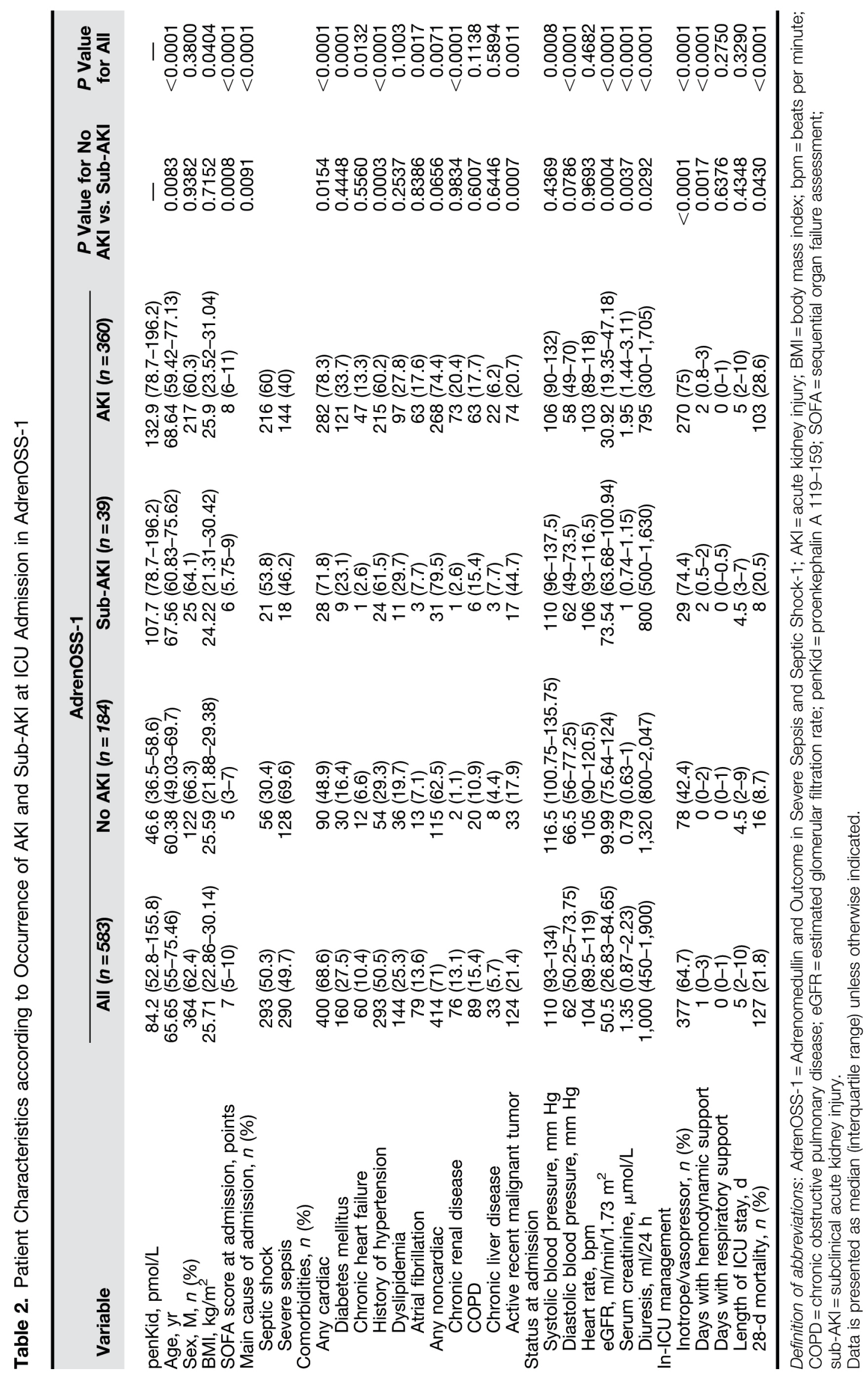



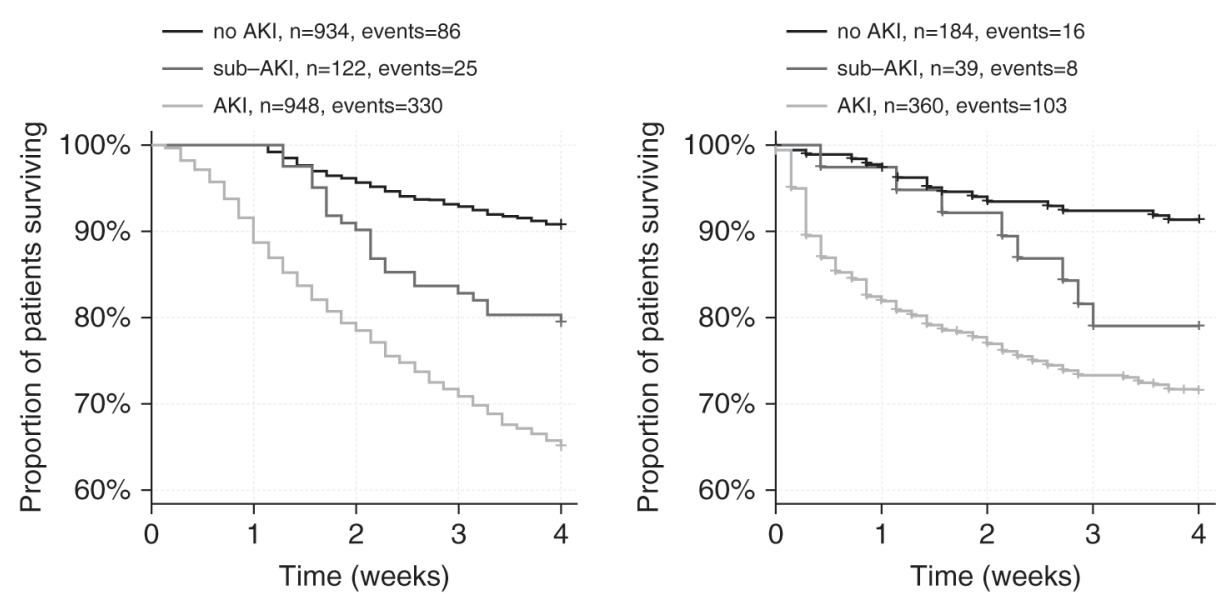

Figure 1. The 28-day mortality Kaplan-Meier curves for patients without acute kidney injury (AKI), subclinical AKI (sub-AKI), and AKI in FROG-ICU (French and European Outcome Registry in ICUs) (left) and AdrenOSS-1 (Adrenomedullin and Outcome in Severe Sepsis and Septic Shock-1) (right). Hazard ratio (95\% confidence interval) for patients with sub-AKI versus no AKI was 2.4 (1.5-3.7) in FROG-ICU and 2.5 (1.1-5.9) in AdrenOSS-1.

subgroups, penKid was superior to both creatinine and diuresis $(P<0.0005$ for all comparisons) (Figure $\mathrm{E} 1$ in the online supplement).

\section{penKid Cutoff Sensitivity to Define Sub-AKI}

In AdrenOSS-1, the optimal cut point for predicting 28-day mortality was $67.2 \mathrm{pmol} / \mathrm{L}$ in patients without $\mathrm{AKI}$ and $62.5 \mathrm{pmol} / \mathrm{L}$ in patients with eGFR $>60 \mathrm{ml} / \mathrm{min} / 1.73 \mathrm{~m}^{2}$. In FROG-ICU, the optimal cut point for predicting 28-day mortality was 49.0

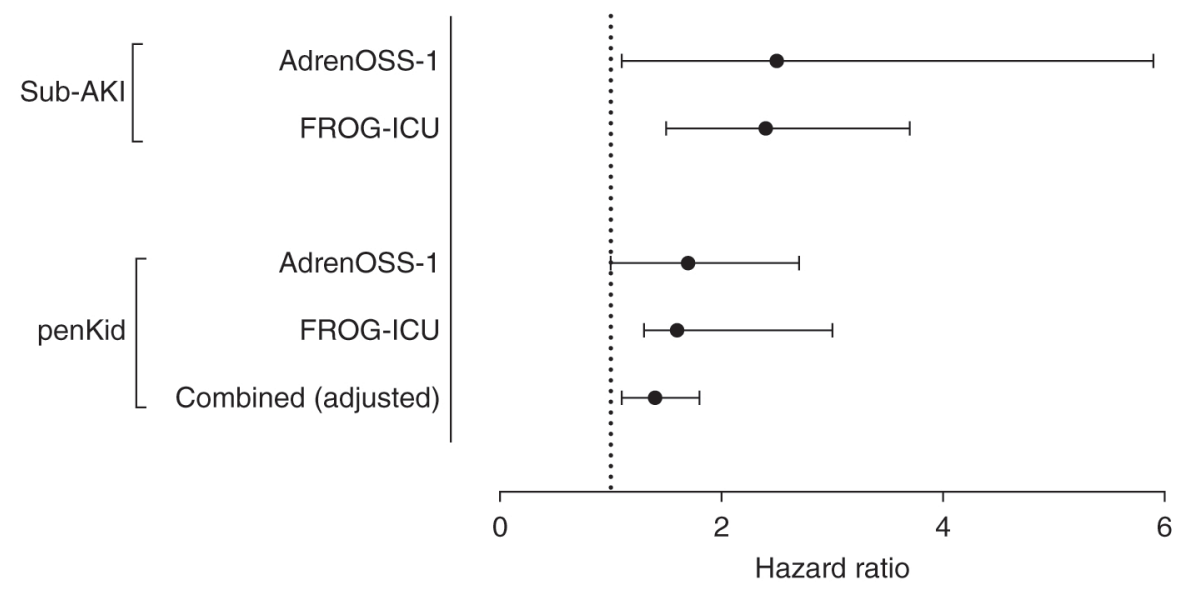

Figure 2. Hazard ratio (HR) of 28-day mortality for subclinical acute kidney injury (sub-AKI) status and (continuous) plasma proenkephalin A 119-159 (penKid) in patients not meeting the Kidney Disease: Improving Global Outcomes definition for AKI. HR for sub-AKI versus non-AKI in AdrenOSS-1 (Adrenomedullin and Outcome in Severe Sepsis and Septic Shock-1) cohort (HR, 2.5 [95\% confidence interval, 1-5.9]) and in FROG-ICU (French and European Outcome Registry in ICUs) cohort (HR, 2.4 [95\% confidence interval, 1.5-3.7]) is shown. Standardized HR for continuous penKid in AdrenOSS-1 study (HR, 1.7 [95\% confidence interval, 1-2.7]) and in FROG-ICU study (HR, 1.6 [95\% confidence interval, 1.3-2.0]), respectively, as well as adjusted standardized HR in the combined cohort (HR, 1.4 [95\% confidence interval, 1.1-1.8]) are also shown.
$(P<0.001)$. In AdrenOSS-1, the need for hemodynamic support within the first week increased from $42.4 \%$ to $74.4 \%$ and $75.0 \%$ in patients with no AKI, sub-AKI, and AKI, respectively $(P<0.001)$. In addition, in AdrenOSS-1, median days on cardiac support within the first week increased from $0(0-2)$ in non-AKI to 2 $(0.5-2)$ in sub-AKI $(P<0.05)$. In patients with AKI, median days on cardiac support was $2(0.8-3)$, comparable to patients with sub-AKI $(P>0.05)$. There was no difference with respect to respiratory support between patients with sub-AKI patients and those without AKI in both cohorts (data not shown).

\section{penKid in Patients with AKI}

For patients with AKI, 28-day survival was $65.2 \%$ ( $n=330$ events) in the FROG-ICU cohort and $71.3 \%$ in the AdrenOSS- 1 cohort ( $n=103$ events). In the FROG-ICU, penKid was associated with 28-day mortality (standardized HR, 1.2 [1.1-1.4], c index, 0.541 [0.509-0.572]; $P=0.0086$ ). Patients with low penKid $(<80 \mathrm{pmol} / \mathrm{L}, 31.3 \%$ of patients with AKI) had slightly lower mortality than patients with elevated penKid, similar to patients without AKI (29.6\% vs. $37.2 \%$, respectively; $P=0.0452$; Figure E2).

In AdrenOSS-1 patients with AKI, penKid was also associated with 28-day mortality (standardized HR, 1.4 [1.1-1.8], c index, 0.590 [0.535-0.644]; $P=0.0088$ ). Patients with low penKid $(<80 \mathrm{pmol} / \mathrm{L}$, $26.1 \%$ ) had a lower mortality than patients with elevated penKid $(18.1 \%$ vs. $32.4 \%$, respectively; $P=0.0058$ ) (Figure E2). In addition, in patients with low penKid, the need for RRT was lower than in patients with high penKid. In low penKid, only $5.3 \%$ received RRT on Day 1, and 9.6\% received RRT at any time during ICU admission.

\section{Discussion}

Our study shows that among ICU patients not meeting the current definition of AKI (i.e., KDIGO), sub-AKI determined by penKid concentration at admission was observed in $12-18 \%$ of patients and was associated with poorer short-term outcome and an increased need for hemodynamic support compared with patients with no AKI. We found that both the risk of death and need for hemodynamic support increased gradually from patients without AKI to those with sub-AKI and those with 


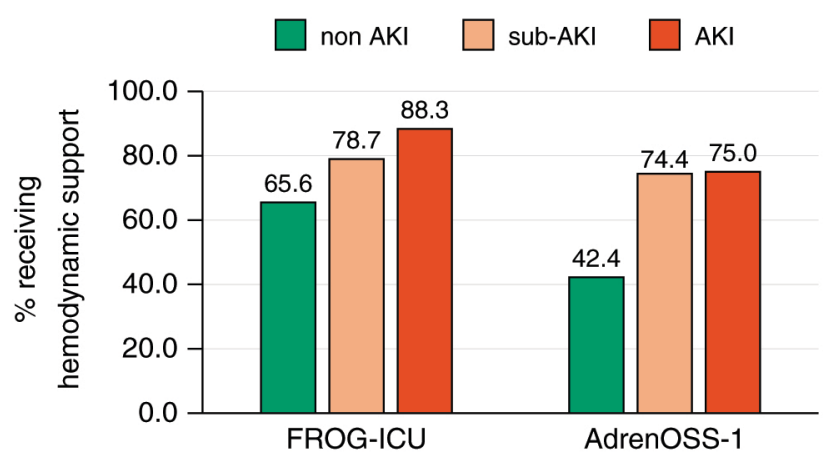

Figure 3. Hemodynamic support in patients without acute kidney injury (AKI), with subclinical AKI (sub-AKI), and with AKI in FROG-ICU (French and European Outcome Registry in ICUs) (left; need for hemodynamic support while in the ICU, $65.6 \%, 78.7 \%$, and $88.3 \%$, respectively; $P<0.001$ ) and AdrenOSS-1 (Adrenomedullin and Outcome in Severe Sepsis and Septic Shock-1) (right; need for hemodynamic support within the first week, $42.4 \%, 74.4 \%$, and $75.0 \%$, respectively; $P<0.001$ ).

AKI. The optimal cut point for mortality prediction was slightly below the 99th percentile of healthy subjects in both studies. Nevertheless, we recommend using the 99th percentile of normal $(80 \mathrm{pmol} / \mathrm{L})$ for the definition of sub-AKI, as values within the normal range may lead to increased false-positive results should the studied population be less severe than ours.

The occurrence of AKI has long been recognized as a strong predictor of mortality in ICU patients (13). The poor sensitivity of Screat to detect AKI has, however, been highlighted (3). Screat increased when a substantial drop of GFR had occurred. Rise of Screat may be blunted in patients with positive fluid balance and/or low muscle mass, two landmarks of critically ill patients. Some episodes of AKI may therefore be unrecognized using Screat. We investigated this later scenario in the current study. Urine output may overcome such limitations, but many physiological factors other than GFR can affect urine output. New biomarkers of AKI have been developed to overcome these limitations (14). The concept of sub-AKI has emerged to designate an episode of AKI unrecognized because of lack of rise in Screat $(5,14)$. In this line, penKid was recently proposed as a biomarker of glomerular function (15-19). penKid is a peptide derived from the precursor of the enkephalin family. Enkephalins are ligands of endogenous opioid receptors widely expressed in the kidney. Increased active enkephalin concentrations are associated with decreased renal function. penKid has been strongly correlated with GFR (19). It is therefore considered as a biomarker of GFR and not a biomarker of renal damage (such as neutrophil gelatinase-associated lipocalin) or kidney stress. This distinction is crucial with respect to the uncoupling between kidney damage and loss of glomerular function.

The incidence of sub-AKI in ICU patients has, however, been largely unexplored. Furthermore, whether sub-AKI in critically ill patients is associated with poor short-term outcome remained elusive. The current data provide evidence that penKid to detect sub-AKI could allow better phenotyping of patients not meeting the current definition of AKI. Potential utility of penKid is underlined by proof of a prognostic impact associated with the levels of this biomarker, which would overcome current AKI definition limitations. The mortality of patients with sub-AKI was higher than patients without sub-AKI and close to patients with AKI. penKid refines the prognosis of patients previously classified as non-AKI patients. penKid therefore allows identification of a subpopulation with an intermediate risk of dying.

Interestingly, patients with sub-AKI required hemodynamic support as frequently as patients with AKI. There might be several reasons for this observation. First, though systemic hemodynamic can certainly impact the occurrence of AKI, the assumption that systemic hemodynamic might be the primary contributor to AKI in critical illness has been repeatedly challenged. Microcirculation disorders, immune and/or inflammatory response, and direct renal toxicity are probably the key contributing factors to AKI in many ICU conditions. In this line, the reasons for ICU admission slightly differed between the group of patients with AKI and sub-AKI. Finally, this might arise from a higher renal function reserve in patients with sub-AKI compared with patients with AKI before ICU admission (20). Lower incidence of comorbidities known to affect the kidney reserve (i.e., diabetes, hypertension, and heart failure) in patients with sub-AKI compared with patients with AKI further reinforces this hypothesis. Larger renal insult would be required to lead to a significant enough drop of GFR to meet the criteria for patients with AKI compared with sub-AKI.

Of note, patients with sub-AKI had slightly higher Screat and lower eGFR compared with those without sub-AKI. These differences might not appear as clinically significant for many physicians because they lie within normal ranges. More importantly, penKid remained associated with mortality after adjustment for Screat, suggesting that penKid refines the risk prediction. Furthermore, a limitation of using low cutoffs for Screat is the rather larger intra- and interpatient variability of Screat. Coefficient of variability of Screat has been reported to be high, therefore introducing an important noise over the signal toward change of renal function when looking at small variations (21-23). The intraassay and intraindividual variability of Screat measurement (including with the enzymatic method) has been reported to significantly impact the diagnosis of, for instance, chronic kidney disease (24). The same issue has been reported with Cystatin C, with intraindividual variance of $8.6 \%$ and interindividual variance of $15.1 \%$ (vs. $14.4 \%$ for Screat). Interlaboratory important variability further adds inaccuracy in estimating small variations (25).

Some patients showed low penKid level despite meeting the definition for AKI. This may arise from different potential scenarios. A first scenario is the late development of AKI, in which penKid was not yet elevated on admission, but AKI developed later during the ICU stay. penKid dosage was performed on admission, whereas serum creatinine samples were taken on a daily basis during the course of ICU stay. Then, penKid could be low at admission and the patient developed AKI later. This is important considering the goal to identify the high-risk population while they are admitted or soon after admission. Late identification of a risk of course hampers all potential attempt to apply preventive or early therapeutic interventions to improve outcomes. The second scenario is 
patients recovering from AKI. Patients were admitted with AKI, but their renal function improved soon after admission. penKid might be seen here as a biomarker of recovery (Screat is still elevated, but while GFR recovers, penKid is found low on admission). This is suggested by the better outcome of patients with AKI presenting with low penKid versus high penKid. We have also discussed this point in the revised version. More research is needed to investigate the impact of serial measurements of penKid to better gain insights into the identification of early recovery in these patients.

Altogether, the results of this study suggest that adding sub-AKI as a new stage of AKI, refining the current definition of AKI, and adding risk stratification and identification of patients with a higher mortality risk are needed.
The current study shows limitations. First, this is an observational cohort study showing an association between sub-AKI and outcome. It remains uncertain whether intervention toward kidney injury may improve outcome even though promising results have been published (26). Second, we used the KDIGO definition to define AKI, and we do not compare penKid to the gold standard for the assessment of kidney function (i.e., inulin clearance) or to biomarkers of renal damage (e.g., neutrophil gelatinase-associated lipocalin, kidney injury molecule-1, livertype fatty acid-binding protein, or TIMP2*IGFBP7). Third, whether or not the combination of penKid and other biomarkers further improve the definition of sub-AKI and its association with outcomes remains to be explored. Fourth, the European origin of the studied population may impede translation of the study results to other populations.

\section{Conclusions}

Sub-AKI detected using penKid occurred in $11.6-17.5 \%$ of patients without AKI and was associated with a risk of death close to patients with AKI and higher than patients without sub-AKI.

Author disclosures are available with the text of this article at www.atsjournals.org.

Acknowledgment: The authors thank Malha Sadoune for technical and logistic assistance for the study. The authors thank the Centre de Recherche Clinique of Lariboisière University Hospital for its support. The authors are particularly grateful to Marie-Céline Fournier, who coordinated organizational aspects of the study.

\section{References}

1. Kellum JA, Lameire N, Aspelin P, et al. Work group membership. Kidney Int 2012;2:1.

2. Legrand M, Kellum JA. Serum creatinine in the critically ill patient with sepsis. JAMA 2018;320:2369-2370.

3. Moledina DG, Parikh CR. Phenotyping of acute kidney injury: beyond serum creatinine. Semin Nephrol 2018;38:3-11.

4. Molitoris BA. Measuring glomerular filtration rate in the intensive care unit: no substitutes please. Crit Care 2013;17:181.

5. Haase M, Kellum JA, Ronco C. Subclinical AKI: an emerging syndrome with important consequences. Nat Rev Nephrol 2012;8:735-739.

6. Caironi P, Latini R, Struck J, Hartmann O, Bergmann A, Bellato V, et al.; ALBIOS Study Investigators. Circulating proenkephalin, acute kidney injury, and its improvement in patients with severe sepsis or shock. Clin Chem 2018;64:1361-1369.

7. Hollinger A, Wittebole X, François B, Pickkers P, Antonelli M, Gayat E, et al. Proenkephalin A 119-159 (penkid) is an early biomarker of septic acute kidney injury: the kidney in sepsis and septic shock (Kid-SSS) study. Kidney Int Rep 2018;3:1424-1433.

8. Marino R, Struck J, Hartmann O, Maisel AS, Rehfeldt M, Magrini L, et al. Diagnostic and short-term prognostic utility of plasma pro-enkephalin (pro-ENK) for acute kidney injury in patients admitted with sepsis in the emergency department. J Nephrol 2015;28:717-724.

9. Mebazaa A, Casadio MC, Azoulay E, Guidet B, Jaber S, Levy B, et al. Post-ICU discharge and outcome: rationale and methods of the The French and euRopean Outcome reGistry in Intensive Care Units (FROG-ICU) observational study. BMC Anesthesiol 2015;15:143.

10. Mebazaa A, Geven C, Hollinger A, Wittebole X, Chousterman BC, Blet $A$, et al.; AdrenOSS-1 study investigators. Circulating adrenomedullin estimates survival and reversibility of organ failure in sepsis: the prospective observational multinational Adrenomedullin and Outcome in Sepsis and Septic Shock-1 (AdrenOSS-1) study. Crit Care 2018;22:354.

11. Kidney disease: improving global outcome (KDIGO) acute kidney injury work group. KDIGO clinical practice guideline for acute kidney injury. Kidney Int Suppl 2012;2:1-138.

12. Gayat E, Hollinger A, Cariou A, Deye N, Vieillard-Baron A, Jaber S, et al.; FROG-ICU investigators. Impact of angiotensin-converting enzyme inhibitors or receptor blockers on post-ICU discharge outcome in patients with acute kidney injury. Intensive Care Med 2018;44:598-605.

13. Bellomo R, Ronco C, Mehta RL, Asfar P, Boisramé-Helms J, Darmon M, et al. Acute kidney injury in the ICU: from injury to recovery: reports from the 5th Paris International Conference. Ann Intensive Care 2017;7:49.
14. Ronco C. Acute kidney injury: from clinical to molecular diagnosis. Crit Care 2016;20:201.

15. Ng LL, Squire IB, Jones DJL, Cao TH, Chan DCS, Sandhu JK, et al.; GREAT Network. Proenkephalin, renal dysfunction, and prognosis in patients with acute heart failure: a GREAT network study. J Am Coll Cardiol 2017;69:56-69.

16. Shah KS, Taub P, Patel M, Rehfeldt M, Struck J, Clopton P, et al. Proenkephalin predicts acute kidney injury in cardiac surgery patients. Clin Nephrol 2015;83:29-35.

17. Matsue Y, Ter Maaten JM, Struck J, Metra M, O'Connor CM, Ponikowski $P$, et al. Clinical correlates and prognostic value of proenkephalin in acute and chronic heart failure. J Card Fail 2017;23: 231-239.

18. Kieneker LM, Hartmann O, Struck J, Bergmann A, Gansevoort RT, Joosten MM, et al. Plasma proenkephalin and poor long-term outcome in renal transplant recipients. Transplant Direct 2017;3: e190.

19. Donato LJ, Meeusen JW, Lieske JC, Bergmann D, Sparwaßer A, Jaffe AS, et al. Analytical performance of an immunoassay to measure proenkephalin. Clin Biochem 2018;58:72-77.

20. Denic A, Glassock RJ, Rule AD. Structural and functional changes with the aging kidney. Adv Chronic Kidney Dis 2016;23:19-28.

21. Hilderink JM, van der Linden N, Kimenai DM, Litjens EJR, Klinkenberg LJJ, Aref BM, et al. Biological variation of creatinine, cystatin C, and eGFR over 24 hours. Clin Chem 2018;64:851-860.

22. Reinhard M, Erlandsen EJ, Randers E. Biological variation of cystatin C and creatinine. Scand J Clin Lab Invest 2009;69:831-836.

23. Joffe M, Hsu CY, Feldman HI, Weir M, Landis JR, Hamm LL; Chronic Renal Insufficiency Cohort (CRIC) Study Group. Variability of creatinine measurements in clinical laboratories: results from the CRIC study. Am J Nephrol 2010;31:426-434.

24. Rowe C, Sitch AJ, Barratt J, Brettell EA, Cockwell P, Dalton RN, et al.; eGFR-C Study Group. Biological variation of measured and estimated glomerular filtration rate in patients with chronic kidney disease. Kidney Int 2019;96:429-435.

25. Lee $\mathrm{E}$, Collier $\mathrm{CP}$, White CA. Interlaboratory variability in plasma creatinine measurement and the relation with estimated glomerular filtration rate and chronic kidney disease diagnosis. Clin J Am Soc Nephrol 2017;12:29-37.

26. Meersch M, Schmidt C, Hoffmeier A, Van Aken H, Wempe C, Gerss J, et al. Prevention of cardiac surgery-associated AKI by implementing the KDIGO guidelines in high risk patients identified by biomarkers: the PrevAKI randomized controlled trial. Intensive Care Med 2017;43: 1551-1561. 